\title{
Crystal structure of bis(trichlorodiethylamidotantalum(V) isopropylate), $\left[\mathrm{TaCl}_{3}\left(\mathrm{C}_{4} \mathrm{H}_{10} \mathrm{~N}\right)\left(\mathrm{C}_{3} \mathrm{H}_{7} \mathrm{O}\right)\right]_{2}$
}

\author{
Stephan Peter BlöB, Jürgen Nuss and Martin Jansen* \\ Max-Planck-Institut für Festkörperforschung, HeisenbergstraBe 1, 70569 Stuttgart, Germany
}

Received January 29, 2009, accepted and available on-line March 11, 2009; CCDC no. 1267/2551

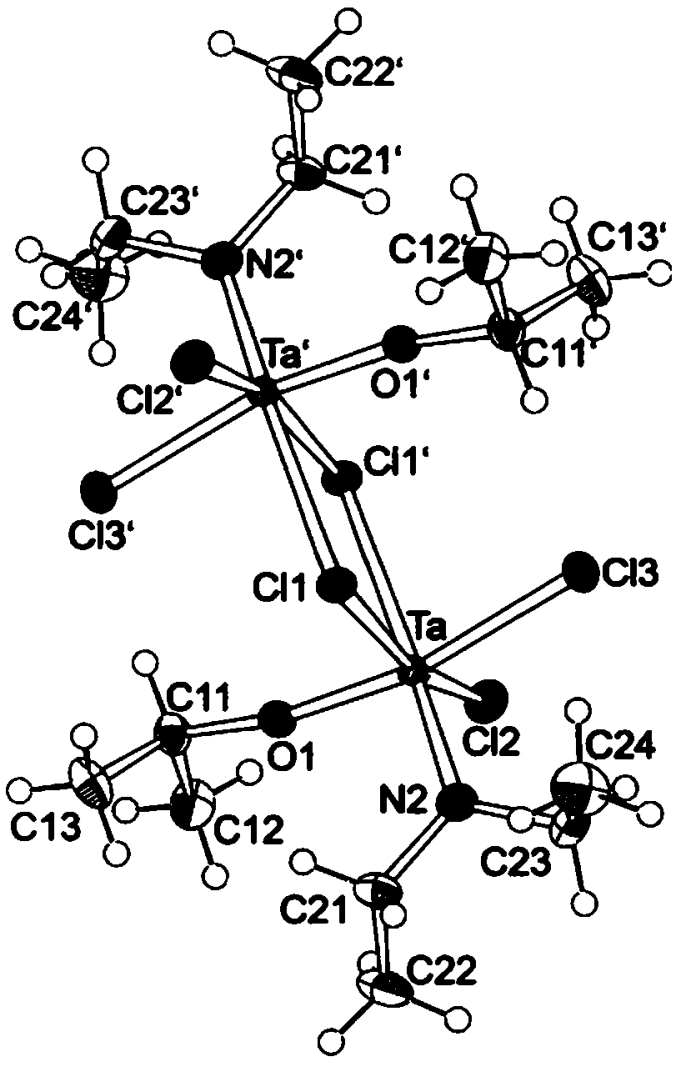

Abstract

$\mathrm{C}_{14} \mathrm{H}_{34} \mathrm{Cl}_{6} \mathrm{~N}_{2} \mathrm{O}_{2} \mathrm{Ta}$, triclinic, $P \overline{1}$ (no. 2), $a=8.0083(6) \AA$,

$b=8.8582(7) \AA, c=10.7360(8) \AA, \alpha=67.809(1)^{\circ}$,

$\beta=86.039(2)^{\circ}, \gamma=69.812(1)^{\circ}, V=660.2 \AA^{3}, Z=1$,

$R_{g t}(F)=0.035, w R_{\text {ref }}\left(F^{2}\right)=0.079, T=100 \mathrm{~K}$.

\section{Source of material}

Following the published prescription [1], $230 \mu \mathrm{L}$ of $\mathrm{Me}_{3} \mathrm{SiNEt}_{2}$ were added dropwise to a solution of $0.42 \mathrm{~g} \mathrm{TaCl}_{5}$ in $20 \mathrm{~mL}$ diisopropyl ether. The yellow solution was allowed to stir at room temperature for $1 \mathrm{~h}$. Afterwards the solution was stored at $-30^{\circ} \mathrm{C}$ for 4 weeks. A piece of honey-yellow crystal was selected from the cold mother solution, transfered in a high viscous oil, and mounted onto a nylon loop for X-ray diffraction.

\section{Discussion}

The title compound was prepared in the course of our studies on single-source precursors for TaON $[1,2]$. The characteristic building unit of the molecular structure is a bi-octahedral dimer, similar to the tantalum pentachloride dimer with two chlorine atoms forming the common edge. One of the equatorial chlorine atoms is substituted by a diethyl amido group whereas the isopropylate group is replacing a chlorine atom from the apical position. The formation of the isopropylate anion can be explained by ether fission under formation of isopropyl chloride. The crystal structure shows no disorder. The Ta-N bond length (1.931(3) $\AA$ ) is in good agreement with those found for other Ta compounds (e.g., $1.912 \AA$ [2]). As expected, the bond length between tantalum and covalently bondend oxygen of the isopropylate anion (1.818(3) $\AA$ ) is much shorter than in tantalum ether compound (2.265(4) $\AA$ ), cf. (diethyl ether)-tetrachlorodimethylamido-tantalum(V), $\mathrm{TaCl}_{4}\left(\mathrm{C}_{2} \mathrm{H}_{6} \mathrm{~N}\right)\left(\mathrm{C}_{4} \mathrm{H}_{10} \mathrm{O}\right)$ [2]. The other distances and angles fit very well the expected values.

Table 1. Data collection and handling.

\begin{tabular}{ll}
\hline Crystal: & $\begin{array}{l}\text { honey-yellow block, } \\
\text { size } 0.30 \times 0.30 \times 0.40 \mathrm{~mm}\end{array}$ \\
Wavelength: & Mo $K_{a}$ radiation $(0.71073 \AA)$ \\
$\mu:$ & $88.99 \mathrm{~cm}^{-1}$ \\
Diffractometer, scan mode: & Bnuker AXS, SMART APEX I, $\omega$ \\
$2 \theta_{\text {max }}:$ & $68.4^{\circ}$ \\
$N(h k l)_{\text {measured, }} N(h k l)_{\text {hnique }}:$ & 19746,5389 \\
Criterion for $\left.I_{\text {obs }}, N(h k)^{\prime}\right)_{\text {gt }}:$ & $I_{\text {ooss }}>2 \sigma\left(I_{\text {obs }}\right), 5187$ \\
$N(\text { param })_{\text {refined: }}$ & 169 \\
Programs: & SHELXS-97 [3], SHELXL-97 [4], \\
& ATOMS [S] \\
\hline
\end{tabular}

Table 2. Atomic coondinates and displacement parameters (in $\AA^{2}$ ).

\begin{tabular}{llllll}
\hline Atom & Site & $x$ & $y$ & $z$ & $U_{\text {iso }}$ \\
\hline H(11) & $2 i$ & $0.324(8)$ & $-0.287(8)$ & $0.889(6)$ & 0.025 \\
H(12A) & $2 i$ & $0.27(1)$ & $-0.181(9)$ & $0.644(8)$ & 0.045 \\
H(12B) & $2 i$ & $0.46(1)$ & $-0.31(1)$ & $0.694(7)$ & 0.045 \\
H(12C) & $2 i$ & $0.43(1)$ & $-0.13(1)$ & $0.639(8)$ & 0.045 \\
H(13A) & $2 i$ & $0.58(1)$ & $-0.14(1)$ & $0.844(8)$ & 0.046 \\
H(13B) & $2 i$ & $0.62(1)$ & $-0.34(1)$ & $0.911(7)$ & 0.046 \\
H(13C) & $2 i$ & $0.53(1)$ & $-0.22(1)$ & $0.987(8)$ & 0.046 \\
H(21A) & $2 i$ & $0.389(8)$ & $0.185(8)$ & $0.792(6)$ & 0.028 \\
H(21B) & $2 i$ & $0.349(8)$ & $0.380(8)$ & $0.744(6)$ & 0.028 \\
H(22A) & $2 i$ & $0.37(1)$ & $0.22(1)$ & $0.558(8)$ & 0.050 \\
H(22B) & $2 i$ & $0.52(1)$ & $0.28(1)$ & $0.586(8)$ & 0.050 \\
H(22C) & $2 i$ & $0.35(1)$ & $0.41(1)$ & $0.514(8)$ & 0.050 \\
H(23A) & $2 i$ & $-0.092(9)$ & $0.546(8)$ & $0.647(6)$ & 0.030 \\
H(23B) & $2 i$ & $0.089(8)$ & $0.564(8)$ & $0.608(7)$ & 0.030 \\
H(24A) & $2 i$ & $-0.05(1)$ & $0.55(1)$ & $0.861(9)$ & 0.058 \\
H(24B) & $2 i$ & $-0.04(1)$ & $0.70(1)$ & $0.734(9)$ & 0.058 \\
H(24C) & $2 i$ & $0.15(1)$ & $0.56(1)$ & $0.839(8)$ & 0.058 \\
& & & & & \\
\hline
\end{tabular}

* Correspondence author (e-mail: M.Jansen@fkf.mpg.de) 
Table 3. Atomic coordinates and displacement parameters (in $\AA^{2}$ ).

\begin{tabular}{|c|c|c|c|c|c|c|c|c|c|c|}
\hline Atom & Site & $x$ & $y$ & $z$ & $U_{11}$ & $U_{22}$ & $U_{33}$ & $U_{12}$ & $U_{13}$ & $U_{23}$ \\
\hline Ta & $2 i$ & $0.03163(2)$ & $0.14174(2)$ & $0.80886(1)$ & $0.01461(7)$ & $0.01340(7)$ & $0.01230(6)$ & $-0.00426(5)$ & $0.00168(4)$ & $-0.00487(5)$ \\
\hline $\mathrm{Cl}(1)$ & $2 i$ & $0.0935(1)$ & $0.1120(1)$ & $1.04334(9)$ & $0.0199(4)$ & $0.0184(3)$ & $0.0151(3)$ & $-0.0093(3)$ & $0.0020(3)$ & $-0.0071(3)$ \\
\hline $\mathrm{Cl}(2)$ & $2 i$ & $-0.0808(1)$ & $0.1266(1)$ & $0.61824(9)$ & $0.0267(4)$ & $0.0242(4)$ & $0.0158(4)$ & $-0.0091(3)$ & $-0.0017(3)$ & $-0.0073(3)$ \\
\hline $\mathrm{Cl}(3)$ & $2 i$ & $-0.2612(1)$ & $0.3301(1)$ & $0.8222(1)$ & $0.0177(4)$ & $0.0199(4)$ & $0.0239(4)$ & $-0.0022(3)$ & $0.0011(3)$ & $-0.0080(3)$ \\
\hline$\alpha(1)$ & $2 i$ & $0.2376(4)$ & $-0.0371(4)$ & $0.8224(3)$ & $0.018(1)$ & $0.017(1)$ & $0.018(1)$ & $-0.005(1)$ & $0.0032(9)$ & $-0.008(1)$ \\
\hline C(11) & $2 i$ & $0.3656(6)$ & $-0.2039(5)$ & $0.8330(4)$ & $0.020(2)$ & $0.020(2)$ & $0.020(2)$ & $-0.003(1)$ & $0.001(1)$ & $-0.010(1)$ \\
\hline$C(12)$ & $2 i$ & $0.3801(7)$ & $-0.2095(7)$ & $0.6934(5)$ & $0.037(2)$ & $0.028(2)$ & $0.021(2)$ & $-0.003(2)$ & $0.002(2)$ & $-0.014(2)$ \\
\hline$C(13)$ & $2 i$ & $0.5401(6)$ & $-0.2271(7)$ & $0.8968(6)$ & $0.017(2)$ & $0.037(2)$ & $0.038(3)$ & $-0.004(2)$ & $-0.002(2)$ & $-0.019(2)$ \\
\hline $\mathbf{N}(2)$ & $2 i$ & $0.1324(5)$ & $0.3235(4)$ & $0.7302(3)$ & $0.023(2)$ & $0.020(1)$ & $0.016(1)$ & $-0.008(1)$ & $0.003(1)$ & $-0.008(1)$ \\
\hline$C(21)$ & $2 i$ & $0.3277(6)$ & $0.2904(6)$ & $0.7251(4)$ & $0.022(2)$ & $0.031(2)$ & $0.023(2)$ & $-0.015(2)$ & $0.005(1)$ & $-0.012(2)$ \\
\hline$C(22)$ & $2 i$ & $0.3914(7)$ & $0.2986(8)$ & $0.5866(5)$ & $0.024(2)$ & $0.051(3)$ & $0.030(2)$ & $-0.019(2)$ & $0.012(2)$ & $-0.017(2)$ \\
\hline$C(23)$ & $2 i$ & $0.0299(6)$ & $0.5135(5)$ & $0.6775(5)$ & $0.028(2)$ & $0.018(2)$ & $0.023(2)$ & $-0.008(2)$ & $-0.002(2)$ & $-0.002(1)$ \\
\hline$C(24)$ & $2 i$ & $0.0165(9)$ & $0.5910(8)$ & $0.7843(7)$ & $0.046(3)$ & $0.033(2)$ & $0.047(3)$ & $-0.015(2)$ & $0.012(3)$ & $-0.025(2)$ \\
\hline
\end{tabular}

\section{References}

1. BlöB, S. P.; Nuss, J.; Jansen, M.: Crystal structure of diethyl ether-tetrachloro-diethylamido-tantalum(V), $\mathrm{TaCl}_{4}\left(\mathrm{C}_{4} \mathrm{H}_{10} \mathrm{~N}\right)\left(\mathrm{C}_{4} \mathrm{H}_{10} \mathrm{O}\right) . \mathrm{Z}$. Kristallogr. NCS 220 (2005) 215-216.

2. Blö̈, S. P.; Nuss, J.; Jansen, M.: Crystal structure of (diethyl ether)tetrachloro-dimethylamido-tantalum(V), $\mathrm{TaCl}_{4}\left(\mathrm{C}_{2} \mathrm{H}_{6} \mathrm{~N}\right)\left(\mathrm{C}_{4} \mathrm{H}_{10} \mathrm{O}\right) . \mathrm{Z}$. Kristallogr. NCS 221 (2006) 209-210.
3. Sheldrick, G. M.: SHELXS-97. Program for the Solution of Crystal Structures. University of Göttingen, Germany 1997.

4. Sheldrick, G. M.: SHELLI-97. Program for the Refinement of Crystal Structures. University of Göttingen, Germany 1997.

5. Dowty, E.: ATOMS. A Complete Program for Displaying Atomic Structures. Version 6.3. Shape Software, Kingsport, Tennessee, USA 2006. 